\section{Prostatakarzinom: GnRH-Blocker senken Testosteron und PSA schnell}

- Die Androgendeprivation ist die Therapie der Wahl bei Patienten mit fortgeschrittenem Prostatakarzinom. Ein wesentlicher Vorteil der GnRH-Blockade sei der schnellere Wirkeintritt ohne initiale Stimulation der GnRHRezeptoren und somit ohne einen Testosteron-Surge, so Prof. Manfred Wirth, Dresden. „Wie bei der Kastration kommt es innerhalb eines Tages zu einem Testosteronabfall in den Kastrationsbereich". Das bestätigen auch die in der 610 Patienten entweder mit dem GnRH-Blocker Degarelix (Firmagon ${ }^{\oplus}$ ) oder mit dem LHRH-Analogon Leuprorelin behandelt wurden. Am Tag 14 beziehungsweise 28 wurde bei Degarelix-Patienten ein PSA-Abfall von $64 \%$ beziehungsweise $85 \%$ verzeichnet im Vergleich zu $18 \%$ und $68 \%$ unter Leuprorelin [Klotz T et al. BJU 2008; 102: 1531-8]. Nach einem Jahr war das PSA-progressionsfreie Überleben in der Degarelix-Gruppe deutlich höher (Hazard Ratio: 0,664; $\mathrm{p}=$ 0,0495).,,34\% der Patienten hatten von Degarelix profitiert", erläuterte Wirth.

In der Extensionsphase der CS21-Studie wurden die Langzeitwirksamkeit von Degarelix untersucht sowie die Effekte eines Wechsels Daten der einjährigen Phase-III-Studie CS21, von Leuprorelin auf den GnRH-Blocker. Die aktuellen Ergebnisse der vierjährigen Nachbeobachtungszeit zeigen, dass die Patienten, die nach zwölf Monaten von Leuprorelin auf Degarelix umgestellten wurden, hinsichtlich des PSA-progressionsfreien Überlebens von dem Switch profitierten. Die Risikorate für einen PSA-Progress wurde signifikant $(p=0,031$ ) reduziert [Crasford ED et al. J Urol 2011; 186: 889-97). Außerdem wurde in der Studie festgestellt, dass Degarelix nicht nur LH (luteinisierendes Hormon), sondern auch FSH (Follikelstimulierendes Hormon) senkt. Die neuen Daten unterstützen die gute Langzeitwirksamkeit und -verträglichkeit von Degarelix. Wegen der guten Datenlage werde in der aktuellen S3-Leitlinie der Deutschen Gesellschaft für Urologie der Einsatz von Degarelix analog zu anderen Formen der Androgendeprivation empfohlen, so Wirth.

Abdol A. Ameri

Satellitensymposium „Generationen verbinden mit wirkungsvollen Therapien bei Enuresis, Testosteronmangelsyndrom und Prostatakarzinom" im Rahmen des 63. DGUKongresses, Hamburg, 15. September 2011 Veranstalter: Ferring, Kiel
Neue Daten zu Sorafenilo

"Mit ihrem umfassenden Entwicklungsprogramm und den zahlreichen klinischen Studien haben Bayer und Onyx die wichtige Rolle von Sorafenib (Nexavar ${ }^{\circledR}$ ) bei der Behandlung des fortgeschrittenen Nierenzellkarzinoms und des inoperablen Leberzellkarzinoms eindeutig belegt", meint Dr. Dimitris Voliotis, Leiter der Globalen Klinischen Entwicklung Onkologie bei Bayer HealthCare.

Auf dem European Multidisciplinary Cancer Congress 2011, der vom 23. bis 27. September in Stockholm stattfand, wurden neue Ergebnisse verschiedener klinischer Studien zu Sorafenib präsentiert. „... Damit unterstreichen die beiden Unternehmen ihr Engagement in der weiteren Erforschung von Nexavar ${ }^{\circledR}$ und seiner Sicherheit und Wirksamkeit bei verschiedenen Tumorarten", erläutert Voliotis.

Nexavar ${ }^{\circledast}$ ist gegenwärtig in mehr als 100 Ländern zugelassen. Bayer und Onyx, behördliche Institutionen, onkologische Arbeitsgruppen sowie einzelne Wissenschaftler untersuchen den Wirkstoff auch in Bezug auf andere Krebsarten.

Nach Informationen von

Bayer, Leverkusen

\section{Zwei Jahrzehnte Mikronährstoffe}

Das auf orthomolekulare Ernährungsmedizin spezialisierte Unternehmen Orthomol schaut in diesem Jahr auf eine 20-jährige Erfolgsgeschichte in Deutschland zurück. Gegründet im Jahr 1991 mit zunächst nur zwei Mitarbeitern, machte das Familienunternehmen die Produktkategorie ergänzende bilanzierte Diät in Deutschland bekannt. Einer Markenumfrage unter 300 Orthomol-Verwendern zufolge schätzen diese besonders die Wirksamkeit, Qualität und Anwenderfreundlichkeit der Produkte. Fast die Hälfte aller Befragten nehmen Orthomol Produkte auf ausdrückliche Empfehlung ihres Arztes ein, weitere $20 \%$ auf Anraten ihres Apothekers.

Orthomol lässt seit 2006 als erstes Unternehmen in seinem Segment die Präparate jährlich nach der ISO 22 000, der höchsten internationalen Norm für Lebensmittelsicherheit, zertifizieren. Von Beginn an setzte das Unternehmen auf den Vertriebskanal Apotheke sowie auf Ärzte und Apotheker als kompetente Empfehler.

Nach Information von

Orthomol, Langenfeld
Nach Informationen von Lilly, Bad Homburg nerinnen Tadalafil bevorzugen, wenn sie mehrere PDE 5-Hemmer kennengelernt hatten. 